\title{
Neuroendocrine responses to a psychosocial stress test for larger groups of participants: comparison of two test exposures
}

\author{
Jakub RajCani ${ }^{1}$, Petra Solarikova ${ }^{1}$, Igor Brezina ${ }^{1}$, Daniela Jezova ${ }^{2}$ \\ ${ }^{1}$ Department of Psychology, Faculty of Arts, Comenius University, Bratislava, Slovakia; ${ }^{2}$ Institute of Experimental Endo- \\ crinology of the Biomedical Research Center, Slovak Academy of Science, Bratislava, Slovakia \\ E-mail:petra.solarikova@uniba.sk
}

\begin{abstract}
Objective. Individual stress tests characterized by social evaluative threat and uncontrollability are known to elicit strong neuroendocrine responses. We tested whether a psychosocial stressor submitted to a larger group of participants (up to 60) may elicit comparable stress responses.

Methods. A total of 59 adult subjects ( 33 women, 26 men) participated in the study, whereas 24 of them suffered from allergy and 35 were healthy. The stress test consisted of a distraction stress task followed by a speech task, in which the participants were randomly subjected to questions related to a topic that they had to prepare as well as arithmetic questions in front of their peers and a committee that responded in standardized and non-supporting manner. State and trait anxiety inventory (STAI) for anxiety state was administrated before and after the test and salivary samples taking. The test was repeated after five months.

Results. The results showed that the shared psychosocial stress application in a larger group of subjects was prosperous. The larger group test (LGST) resulted in an enhanced subjectively experienced stress and an intensive sympathetic nervous system activation, reflected by elevated salivary alpha-amylase activity and the heart rate. The cortisol increment after exposure to the stress test was not significant. Repeated exposure to the test failed to reproduce the original stress responses with exception of the heart rate rise.

Conclusions. In a larger group of subjects, the psychosocial stress test did elicit stress responses similar to the individual stress tests. Our data indicate that the above-mentioned stress test is apparently not an appropriate approach for the repeated use.
\end{abstract}

Key words: stress protocol, groups, HPA axis, sympathetic nervous system

In the real life, most of the stressors are emotional or psychosocial in nature. Therefore, the mental stress tests are also needed under laboratory settings. The most intensive neuroendocrine response has been observed in laboratory stress tasks characterized by social evaluative threat and uncontrollability (Dickerson and Kemmeny 2004; Makatsori et al. 2004; Buzgoova et al. 2020). One of the most commonly used is the Trier Social Stress Test (TSST) (Kirschbaum et al. 1993) and its modification, the Simu- lated Public Speech one (Jezova et al. 2004). While the reliability of the TSST to elicit stress responses has many times been replicated (Allen et al. 2017), the protocol is time consuming and the setting may vary between participants. Certain attempts have been made to modify the test to make it more effective and a protocol of TSST for groups (TSST-G) has been established allowing simultaneous investigation in groups with 4-6 participants (Von Dawans et al. 2011; Boesch et al. 2014). 
In the present study, we aimed to develop a psychosocial stress test that could be performed in a larger group of subjects (up to 60 participants) enabling a simultaneous testing within a single session (large group stress test - LGST). Such test would enable a rapid testing under the same conditions for all subjects. The stressfulness of the procedure was evaluated by a state anxiety questionnaire and neuroendocrine response by salivary cortisol concentration, salivary alpha-amylase (sAA) activity, and the heart rate level. To examine the possibility of repetitive use of LGST, the volunteers were exposed to two LGST five months apart, aiming to find out whether the repeated LGST may elicit effects comparable to the first exposure.

\section{Subjects and methods}

Subjects. The research sample consisted of 59 participants (33 females, 26 males); age 18-29 years (mean \pm S.D.; $20.14 \pm 2.00$ years). During the recruitment, 24 of available subjects were allergic (either atopic dermatitis, allergic rhinitis or their combination). Allergy was considered as a possible interfering factor in the statistical analysis. All participants had no other diseases and they did not take any corticosteroid medication. Some of the allergic subjects treated with antihistamines skipped their treatment on the day of measurement. Written consent was obtained from all the participants after being acquainted by all the research procedures used. The study was approved by the Ethical committee of the Trnava city Self-Governing Region (Trnava, Slovakia).

Due to missing material, i.e. not enough saliva in the samples, technical difficulties with ECG probes, etc., only 55 participants were analyzed for salivary cortisol, 56 for sAA activity, 46 for heart rate, and 57 for state anxiety (State-Trait Anxiety Inventory STAI).

Procedure. The participants were exposed to two LGST tests 5 months apart. The stress test was administered from 14:00-16:00 p.m. In both testing terms, the task was analogous and differed only in the order of its parts. LGST was realized in a standard lecture room with all 59 participants at once as well as two experimenters. The participants were seated with one free seat between them. Upon entering the room, the participants received a pack of four salivettes (two for each sample), a set of STAI questionnaires, and paper sheets in an envelope. The first salivary sample was taken after the participants were seated. Their heart rate recording devices were applied and switched on. After taking the sample, the participants completed the first STAI questionnaire and waited for another 5 minutes (adaptation phase). LGST consisted of two parts. In the initial part, the distraction stress task lasting 5 minutes was performed. The participants had to go through the sample text as fast as possible while circling all consonants in the text. The instruction changed upon different hearings: a high tone meant to cross out vowels in the text and a low tone to go back to the original. The instruction changed back and forth every 10-20 seconds in an irregular fashion. Moreover, the participants after encountering a meaningless sentence in the text, they should underline the seventh letter in that sentence, regardless of the tone heard.

The subsequent part of the LGST was the speech test with preparation, which was based on TSST-like protocol. First, the participants were given 5 minutes to prepare their speech on a given topic, they could write down their notes; however, these could not be used during the presentation (Hlavacova et al. 2017; Solarikova et al. 2018). The participants presented their speech to a three-person committee in front of their peers. Similar to the original TSST protocol, the committee answered in a standardized, nonsupporting manner. After the preparation, the participants were given a proxy task (filling the Bourdon attention test) to solve as quickly as possible, while at the same time, they were called out in the randomized order and asked arithmetic questions by the committee. Every participant was asked to step out and present their speech. The stress test was based on unpredictability, as the same participant could be asked multiple times, and social evaluative situation. This part lasted 25 minutes, after which the second salivary sample was taken and the second STAI filled up.

The same test was applied after approximately 5 months. During the second exposure to the test, the order of its parts was reversed, starting with preparation and verbal task followed by the distraction stress.

Dependent variables. Saliva was collected via sterile salivettes (Sallivette, Sarstedt, UK) and kept frozen until analyzed. Salivary cortisol concentration and sAA activity were determined using a commercially available kinetic reaction assay (Salimetrics, Suffolk, UK) and enzyme-linked immunosorbent assay (IBL International, Hamburg, Germany), respectively. The heart rate was averaged from two 5 min recordings using FAROS 90 portable ECG device. The first recording was done during the adaptation phase, while the second one at the end of the speech task. STAI-S questionnaire for the anxiety state was presented to participants at the beginning and at the end of the protocol. 
Statistical analysis. Data were analyzed in JASP (JASP Team, 2020) via repeated measures ANOVA model with repeated factor of measurements before and after the test (two salivary samples for cortisol and sAA, two 5-minute heart rate measurements before and during the test, two repetitions of STAI-S inventory). Model also included groups based on the presence of allergy as a covariate. Dependent variables including cortisol, sAA, heart rate, and state anxiety were analyzed separately. Cortisol and sAA measurements were $\log 10$ transformed to adjust for positive skew. Confidence intervals were calculated in R package MBESS (Kelley 2020).

Because LGST was done in two exposures, the same models were calculated with data from the second test. Finally, for comparison of LGST1 and LGST2, the average increases in each variable were calculated and compared via paired $\mathrm{t}$-test.

\section{Results}

As shown in Figure 1 and Table 1, the first LGST induced only a small salivary cortisol increase for the overall sample. This main effect was not statistically significant $\left(\mathrm{F}_{(1,52)}=1.26, \mathrm{p}=0.268, \eta_{p}^{2}=0.03\right)$. In sAA activity, we found a strong increase after LGST $\left(\mathrm{F}_{(1,52)}=16.07, \mathrm{p}<0.001, \eta_{p}^{2}=0.24, \mathrm{CI}_{90}=0.08-0.38\right)$. Even larger rise was observed in the heart rate $\left(\mathrm{F}_{(1,44)}=70.12\right.$, $\left.\mathrm{p}<0.001, \eta_{p}^{2}=0.61, \mathrm{CI}_{90}=0.45-0.71\right)$. The perception of stress and the stressfulness of the LGST were reflected by a subjectively reported anxiety increase $\left(\mathrm{F}_{(1,55)}=8.24, \mathrm{p}=0.006, \eta_{p}^{2}=0.13, \mathrm{CI}_{90}=0.02-0.27\right)$.

A covariate in the present model was allergy of the participants. However, no statistically significant main effect was found in any of the tested variables (all p-values $>0.2$, all $\eta_{p}^{2}$-values $<0.03$ ) and neither the interactions with time factor were significant (all p-values $>0.1$, all $\eta_{p}^{2}$-values $<0.04$ ). Figure 1 summarizes the stress responses elicited by the LGST protocol in the whole sample.

When the same protocol was repeated after 5 months, the stress responses of the participants were lower than in the first test. Cortisol remained unchanged during the test $\left(\mathrm{F}_{(1,53)}=2.22, \mathrm{p}=0.142\right.$, $\left.\eta_{p}^{2}=0.04\right)$. Similarly, sAA activity did not change $\left(\mathrm{F}_{(1,54)}=0.58, \mathrm{p}=0.451, \eta_{p}^{2}=0.01\right)$. The heart rate increased during the test, but this increase reached only the half value of the first test $\left(F_{(1,49)}=13.26\right.$, $\left.\mathrm{p}=0.001, \eta_{p}^{2}=0.21, \mathrm{CI}_{90}=0.06-0.36\right)$. The self-reported anxiety remained unchanged $\left(\mathrm{F}_{(1,53)}=2.61, \mathrm{p}=0.112\right.$, $\left.\eta_{p}^{2}=0.05\right)$.

With respect to the covariate of allergy, no statistically significant main effect was found in any of the tested variables (all $\mathrm{p}$-values $>0.15$, all $\eta_{p}^{2}$-values $<0.04)$. Similarly, neither of the interaction effects was significant (all p-values $>0.2$, all $\eta_{p}^{2}$-values $<0.03$ ).

To evaluate the differences between the two exposures in the LGST protocol, increments were calculated (as after test value - baseline value) for all variables and these were compared between the two test exposures using paired $\mathrm{t}$-tests. With the heart rate exception, the increments of all the variables were significantly lower during the repeated test compared to those induced by the first exposure (Table 1).

\section{Discussion}

The results of this study demonstrate that it is feasible to induce a psychosocial stress situation in a larger group of subjects (up to 60 participants). The stressfulness of the LGST protocol is based on an unpredictability and social evaluative situation. The

Table 1

Comparison of stress responses in the two repetitions of LGST divided by 5 months

\begin{tabular}{|c|c|c|c|c|c|c|c|c|c|}
\hline & & \multirow{2}{*}{$\begin{array}{c}\text { Mean } \\
\text { increase }\end{array}$} & \multirow{2}{*}{ SE } & \multirow{2}{*}{$\mathrm{t}$} & \multirow{2}{*}{ df } & \multirow{2}{*}{ p-value } & \multirow{2}{*}{ Cohen d } & \multicolumn{2}{|c|}{$95 \mathrm{CI}$ for $\mathrm{d}$} \\
\hline & & & & & & & & lower & upper \\
\hline \multirow{2}{*}{ Salivary cortisol } & Test 1 & 0.66 & 0.39 & \multirow{2}{*}{2.15} & \multirow{2}{*}{50} & \multirow{2}{*}{0.037} & \multirow{2}{*}{0.30} & \multirow{2}{*}{0.02} & \multirow{2}{*}{0.58} \\
\hline & Test 2 & -0.28 & 0.27 & & & & & & \\
\hline \multirow{2}{*}{ sAA } & Test 1 & 24.88 & 4.85 & \multirow{2}{*}{5.49} & \multirow{2}{*}{51} & \multirow{2}{*}{$<0.001$} & \multirow{2}{*}{0.76} & \multirow{2}{*}{0.45} & \multirow{2}{*}{1.07} \\
\hline & Test 2 & -4.58 & 4.50 & & & & & & \\
\hline \multirow{2}{*}{ Heart rate } & Test 1 & 11.12 & 1.27 & \multirow{2}{*}{1.74} & \multirow{2}{*}{39} & \multirow{2}{*}{0.089} & \multirow{2}{*}{0.28} & \multirow{2}{*}{-0.04} & \multirow{2}{*}{0.59} \\
\hline & Test 2 & 5.50 & 1.50 & & & & & & \\
\hline \multirow{2}{*}{ STAI score } & Test 1 & 4.35 & 1.36 & \multirow{2}{*}{3.43} & \multirow{2}{*}{52} & \multirow{2}{*}{0.001} & \multirow{2}{*}{0.47} & \multirow{2}{*}{0.19} & \multirow{2}{*}{0.75} \\
\hline & Test 2 & -1.84 & 1.10 & & & & & & \\
\hline
\end{tabular}

The table shows mean increments of cortisol, sAA, heart rate and STAI score in both tests as well as paired comparison of the two tests. Abbreviations: LGST - large group stress test; STAI - State-Trait Anxiety Inventory; sAA - salivary alpha-amylase. 


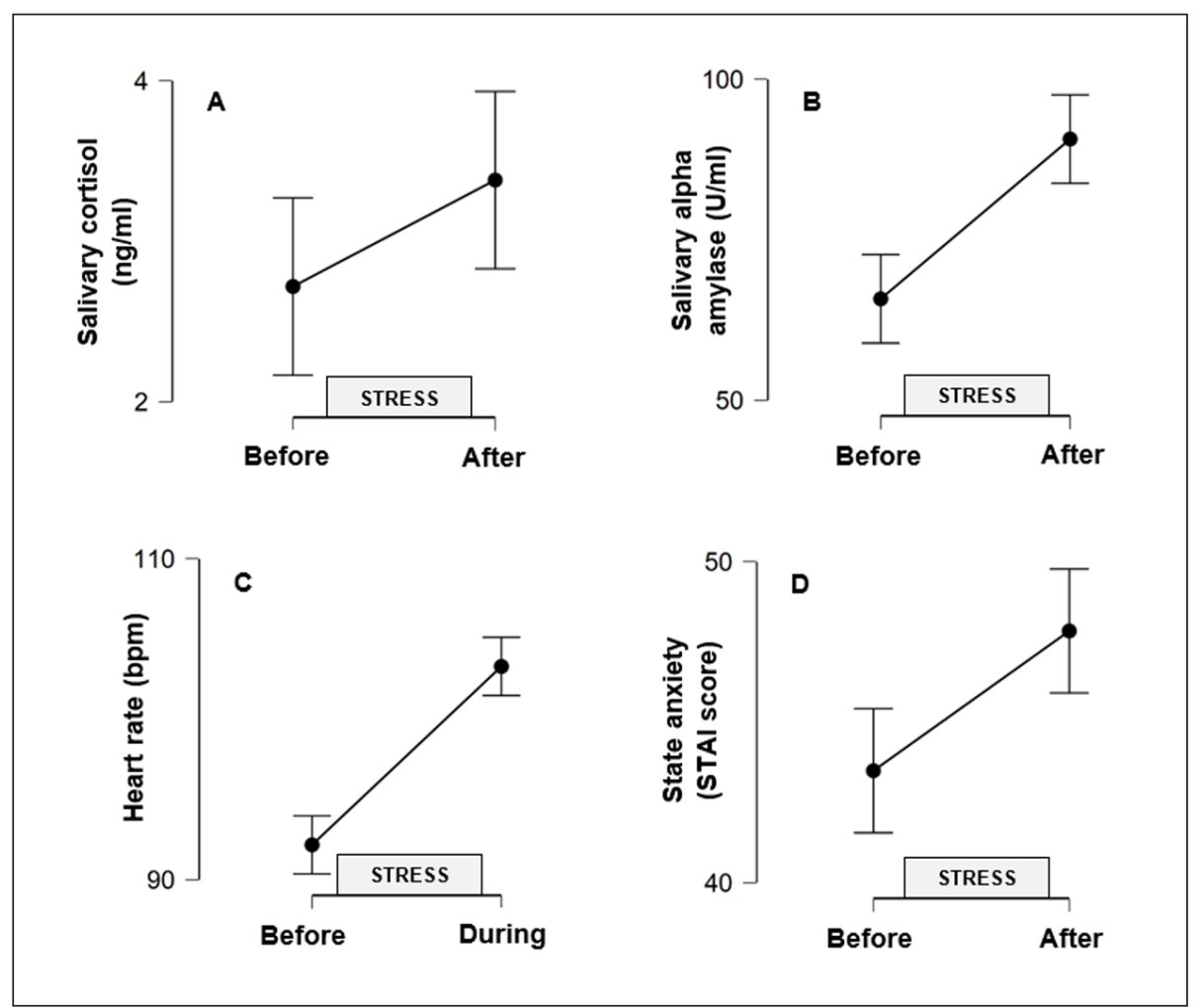

Figure 1. Stress responses in each of the measured variables A) cortisol; B) salivary alpha-amylase; C) heart rate; D) state anxiety in the first large group stress test (LGST). Data are presented as means and $95 \%$ confidence intervals of means in absolute units. Increases in salivary alphaamylase, heart rate and state anxiety were statistically significant $(\mathrm{p}<0.05)$.

LGST resulted in an enhanced subjectively experienced stress and an intensive sympathetic activation as reflected by the elevated sAA activity and the heart rate level. The cortisol increment after the first LGST exposure failed to reach statistical significance, but it was significantly higher compared to that seen after the second exposure. The LGST is apparently not an appropriate approach for the repeated testing.

The observed sympathetic activation is consistent with the general knowledge regarding the effects of mental stressors. A new aspect is that the LGST is accompanied by a "shared" psychosocial stress condition as the variability of the individual responses was not high. The weak cortisol response at the end of the LGST is unlikely to be due to the test low stressfulness. The peak response in salivary cortisol in individual TSST or public speech tests appeared 15 minutes after the test cessation (Makatsori et al. 2004; Jezova et al. 2016). However, it cannot be excluded that an increase would be found when an additional sample will be analyzed. As a marginal sign of the hypothalamic-pituitary-adrenocortical axis activation can be considered a higher cortisol increment in response to the first compared to the second LGST exposure.

No effect of allergy on the neuroendocrine response was revealed by the present analysis, which is supposedly in contrast to previously published data on the reduced sAA and/or cortisol responses to psychosocial stress tests (Buske-Kirschbaum et al. 2010; Hlavacova et al. 2017) or awakening (Rajcani et al. 2019) in allergy. However, the present study was not aimed to investigate the effect of allergy. No efforts were taken to matching the allergic participants with healthy subjects by the number, age, body mass index, sex or menstrual cycle phase. Therefore, the lack of the allergy effect is not surprising and allergy can also be neglected in the evaluation of the neuroendocrine stress response in the large group of participants investigated.

The obtained data show substantially weakened neuroendocrine response to the second exposure to LGST with a significant increase of the heart rate only. Although a habituation of the stress responses 
is commonly seen as a limitation of psychosocial stress tests (Allen et al. 2017), the literature data have suggested that TSST can repeatedly be performed after approximately 10 weeks (Petrowski et al. 2012). However, it was reasonable to expect that the increase in the heart rate during a public speech test was absent in artists, who get adapted to the psychosocial stress of public speech in the real life (Jezova et al. 2016). As the psychosocial stress tests may affect the cognitive functions, the differences mentioned above may be explained by different effects on the memory (Wolf 2019).

In conclusion, the presented LGST is a suitable test inducing a shared psychosocial stress condi- tion for a larger group of subjects, i.e. more than 60 ones. Another advantage of LGST is that besides of only two-time intervals measurements, an additional extra sample, for obtaining a broad neuroendocrine response, can be added on the end of the test. However, repeated exposure to LGST after 5 months did not reproduce the original stress responses, except the heart rate rise.

\section{Acknowledgements}

The study was supported by grant of the Slovak Research and Development Agency APVV-17-0451 and VEGA grants 1/0739/17 and 1/0757/19.

\section{References}

Allen AP, Kennedy PJ, Dockray S, Cryan JF, Dinan TG, Clarke G. The Trier Social Stress Test: principles and practice. Neurobiol Stres 6, 113-126, 2017.

Boesch M, Sefidan S, Ehlert U, Annen H, Wyss T, Steptoe A, La Marca R. Mood and autonomic responses to repeated exposure to the Trier Social Stress Test for groups (TSST-G). Psychoneuroendocrinology 43, 41-51, 2014.

Buske-Kirschbaum A, Ebrecht M, Hellhammer DH. Blunted HPA axis responsiveness to stress in atopic patients is associated with the acuity and severeness of allergic inflammation. Brain Behav Immun 24, 1347-1353, 2010.

Buzgoova K, Balagova L, Marko M, Kapsdorfer D, Riecansky I, Jezova D. Higher perceived stress is associated with lower cortisol concentrations but higher salivary interleukin-1beta in socially evaluated cold pressor test. Stress 23, 248-255, 2020.

Dickerson SS, Kemeny ME. Acute stressors and cortisol responses: a theoretical integration and synthesis of laboratory research. Psychol Bull 130, 355-391, 2004.

Hlavacova N, Solarikova P, Marko M, Brezina I, Jezova D. Blunted cortisol response to psychosocial stress in atopic patients is associated with decrease in salivary alpha-amylase and aldosterone: Focus on sex and menstrual cycle phase. Psychoneuroendocrinology 78, 31-38, 2017.

Jezova D, Makatsori A, Duncko R, Moncek F, Jakubek M. High trait anxiety in healthy subjects is associated with low neuroendocrine activity during psychosocial stress. Progr Neuro Psychopharmacol Biol Psychiatr 28, 1331-1336, 2004.

Jezova D, Hlavacova N, Dicko I, Solarikova P, Brezina I. Psychosocial stress based on public speech in humans: is there a real life/laboratory setting cross-adaptation? Stress 19, 429-433, 2016.

Kelley K. The MBESS R Package. 2020. Retrieved from: https://cran.r-project.org/web/packages/MBESS/index.html. Kirschbaum C, Pirke KM, Hellhammer DH. The 'Trier Social Stress Test'--a tool for investigating psychobiological stress responses in a laboratory setting. Neuropsychobiology 28, 76-81, 1993.

Makatsori A, Duncko R, Moncek F, Loder I, Katina S, Jezova D. Modulation of neuroendocrine response and nonverbal behavior during psychosocial stress in healthy volunteers by the glutamate release-inhibiting drug lamotrigine. Neuroendocrinology 79, 34-42, 2004.

Petrowski K, Wintermann GB, Siepmann M. Cortisol response to repeated psychosocial stress. Appl Psychophysiol Biofeedback 37, 103-107, 2012.

Rajcani J, Solarikova P, Buzgoova K, Brezina I, Jezova D. Patients with atopy exhibit reduced cortisol awakening response but not cortisol concentrations during the rest of the day. Immunol Res 67, 176-181, 2019.

Solarikova P, Brezina I, Turonova D, Rajcani J, Mlyncekova S. Long-term monitoring of heart rate variability and state anxiety in allergic patients: comparison of four stress protocols. Cesk Psychol 62, 258-271, 2018.

Von Dawans B, Kirschbaum C, Heinrichs M. The Trier Social Stress Test for groups (TSST-G): A new research tool for controlled simultaneous social stress exposure in a group format. Psychoneuroendocrinology 36, 514-522, 2011.

Wolf OT. Memories of and influenced by the Trier Social Stress Test. Psychoneuroendocrinology 105, 98-104, 2019. 